\title{
Beyond the Standard Model, Experimental Summary
}

\author{
R. A. McPherson ${ }^{\mathrm{a}}$

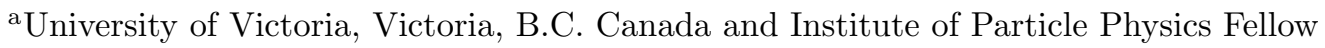

An overview of experimental results in searches for physics beyond the Standard Model is presented. It is impossible to cover all topics in this field, so a set of examples is used to highlight the scope and breadth of the results. Selected topics include searches for compositeness, flavour changing neutral currents, SUSY, exotic Higgs particles, low scale gravity in extra dimensions, and non commutative geometry. Current results are presented from the LEP, Tevatron Run I, and HERA I experiments. No convincing evidence for physics beyond the Standard Model has been observed. Prospects for ongoing and upcoming experiments are discussed.

\section{Introduction}

The field of searches for physics beyond the Standard Model (SM) is one of the most active in all of High Energy Physics, in both experiment and theory. There are direct searches for the production of new particles, and also indirect searches for deviations in cross-sections of Standard Model processes which could be caused by new physics. The field is driven by the impression that the SM is incomplete, and that there may be new physics which explains the observed particle generation structure, quantum numbers, and large number of free SM parameters. Perhaps the most severe problem for the SM is the problem of the hierarchy of energy scales between the electroweak (EW) and gravitational interactions. If one believes that there is no new physics between the EW and gravitational scales, the fine tuning of the SM Higgs particle mass due to particle loop corrections is extreme. Many models of new physics have been proposed to address these issues.

The experimental sensitivity to most realized models of new physics is dominated by results from the three high-energy colliders, LEP, the Tevatron, and HERA. While it is obvious that the mass reach for direct particle production increases with beam energy, in many cases the contributions of new physics to indirect searches also increase with the beam energy and thus higher energy data rapidly supersedes lower energy re- sults.

The Large Electron Positron Collider (LEP) ran from 1989-2000 with four experiments (ALEPH, DELPHI, L3 and OPAL). In the "LEP I" phase, a data set of about $180 \mathrm{pb}^{-1}$ was collected by each experiment at centre-ofmass energies, $\sqrt{s}$, near $M_{\mathrm{Z}}$. Both direct searches and precision electro-weak measurements from LEP I severely constrain any new physics with a scale below about $M_{\mathrm{Z}} / 2$, unless the new physics decouples from the Z-boson. In the "LEP II" phase, a data set of about $700 \mathrm{pb}^{-1}$ was collected by each experiment at centre-of-mass energies from 130-209 GeV, with about $250 \mathrm{pb}^{-1}$ having $\sqrt{s}>200 \mathrm{GeV}$. The LEP experiments reported many new results to ICHEP02, and are in the process of finalizing many of their searches for new physics. The LEP results are in many cases the most constraining on models of new physics, and are likely to remain so for new physics with predominantly EW-strength couplings until the CERN Large Hadron Collider, the LHC, begins operation.

The Tevatron proton-antiproton collider at Fermilab ran during its "Run I" phase from 19891994 with two experiments (CDF and D0). Each experiment accumulated approximately $100 \mathrm{pb}^{-1}$ at $\sqrt{s} \simeq 1.8 \mathrm{TeV}$. The upgraded experiments are now in the "Run IIa" phase, hoping to each accumulate about $2 \mathrm{fb}^{-1}$ at $\sqrt{s} \simeq 2 \mathrm{TeV}$ over the next two years, and will then move on to "Run IIb" with another $\sim 15 \mathrm{fb}^{-1}$ per experiment before 
the LHC era begins. The Run I data set is in many cases the most constraining on models with new, strongly interacting particles such as scalar quarks or lepto-quarks. With the Run II data, CDF and D0 will also be rather competitive with LEP in searches for particles with electroweakstrength production if they have distinctive decay products including energetic photons or leptons which allow for efficient triggers and clean separation from the background processes.

The HERA facility collides protons with electrons or positrons at $\sqrt{s}=300-320 \mathrm{GeV}$. In the first phase, "HERA I", the H1 and ZEUS experiments each accumulated approximately $100 \mathrm{pb}^{-1}$ of data. The facility is now running in the higher luminosity "HERA II" phase, and hope to accumulate about $1 \mathrm{fb}^{-1}$ of data running until about 2006. The HERA data is particularly sensitive to new particles with significant couplings to first generation leptons and quarks, such as some leptoquarks, R-Parity violating squarks, or possibly anomalous FCNC that could allow a strong topup quark coupling.

\section{Compositeness and anomalous FCNC couplings}

Compositeness, or fermion substructure, is one of the most enduring ideas of possible physics beyond the Standard Model. It could provide an intuitive explanation for the hierarchical fermion generation structure, and a connection between the quarks and the leptons. Compositeness, or other possible new physics, could also lead to anomalous couplings between the different fermion generations. Such effects might be observed in Standard Model rare or forbidden processes like Flavour Changing Neutral Currents (FCNC). While FCNC effects have been searched for with high precisions in lighter particle decays, there remains considerable experimental scope for FCNC in the top quark couplings.

A sensitive probe of compositeness is the search for anomalous 4-fermion contact interactions [1] in processes such as $\mathrm{e}^{+} \mathrm{e}^{-} \rightarrow \ell^{+} \ell^{-}, \mathrm{e}^{+} \mathrm{e}^{-} \rightarrow \mathrm{q} \overline{\mathrm{q}}$, $\mathrm{q} \overline{\mathrm{q}} \rightarrow \ell^{+} \ell^{-}$and eq $\rightarrow$ eq. These processes also constrain the masses and couplings of new vector bosons. LEP, HERA and the Tevatron experi- ments have all searched for 4 -fermion contact interactions beyond the Standard Model, but found no significant signal in any channel. Assuming electromagnetic strength couplings, 4-fermion contact interactions exclude new physics at scales below about $1-10 \mathrm{TeV}$, depending on the precise nature of the coupling. While this is constraining, the 4-fermion contact interaction formalism neglects new particle propagator effects, and is therefore not valid for particles within the kinematic reach of the colliders. In that region, even including particle propagator effects, the direct searches dominate the sensitivity to new physics.

\subsection{Excited fermions}

One natural consequence of fermion compositeness would be the existence of excited states of the known quarks and leptons, $\mathrm{q}^{*}$ and $\ell^{*}$, which could be produced at colliders and then decay to their Standard Model partners via the emission of a vector boson. Most experiments use the phenomenological model described in $[2,3]$ to guide their searches and interpret their results. This model has, in addition to the excited fermion masses, three couplings $(S U(3)$ coupling $f_{s}, S U(2)$ coupling $f$ and $S U(1)$ coupling $f^{\prime}$ ) and one scale $(\Lambda)$ as free parameters. The classic signature of excited leptons, a peak in the leptonphoton mass spectrum from the decay $\ell^{*} \rightarrow \ell \gamma$, would be a spectacular signature for new physics. An example of the search for the production of excited electrons with electromagnetic decays from ZEUS in the process ep $\rightarrow \mathrm{e}^{*} \mathrm{X}$ with $\mathrm{e}^{*} \rightarrow \mathrm{e} \gamma$ is shown in Figure 1 [4].

Alternatively, excited electrons could be observed indirectly in the process $\mathrm{e}^{+} \mathrm{e}^{-} \rightarrow \gamma \gamma$, where contributions from $t$-channel excited electron exchange would modify the Standard Model differential cross-section. The effect of excited electrons on the $\mathrm{e}^{+} \mathrm{e}^{-} \rightarrow \gamma \gamma$ process has recently been calculated [5] allowing a direct comparison of the constraints from this processes to those from direct searches. An example of a fit to excited electron contributions in $\mathrm{e}^{+} \mathrm{e}^{-} \rightarrow \gamma \gamma$ from OPAL is shown in Figure 2 [6].

Combining all constraints on excited electrons, and assuming as an example the coupling relation $f=f^{\prime}$, results in the constraints shown in 


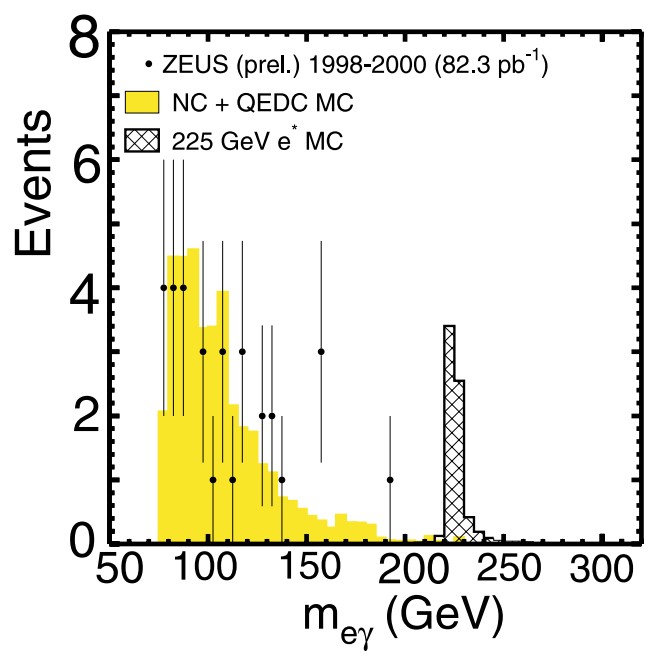

Figure 1. The e $\gamma$ invariant mass distribution from ZEUS 1998-2000 data. The points with error bars are the data, and the shaded region is the SM background expectation. The hatched histogram is the expectation for a $225 \mathrm{GeV}$ excited electron with the decay $\mathrm{e}^{*} \rightarrow \mathrm{e} \gamma$, with arbitrary normalization.

Figure 3. There is a direct constraint on the $\mathrm{e}^{*}$ mass from the search for the process $\mathrm{e}^{+} \mathrm{e}^{-} \rightarrow$ $\mathrm{e}^{*} \mathrm{e}^{*} \rightarrow$ e $\gamma \mathrm{e} \gamma$ from the DELPHI [7] and OPAL [8] experiments, excluding masses less than about $103 \mathrm{GeV}$. For larger masses, searches from HERA and LEP constrain the coupling for different excited electron masses [6-9].

\subsection{Leptoquarks}

Another consequence of common substructure of quarks and leptons could be the existence of particles with the quantum numbers of both, leptoquarks (LQ). Leptoquarks could, in fact, exist in many models of new physics. The most commonly used leptoquark model is from Buchmüller-Rückl-Wyler [10]. It allows only a specific set of leptoquark quantum numbers, particularly the fermion number $F$ (the sum of the lepton and baryon number) and the electric charge. The model also restricts the branching

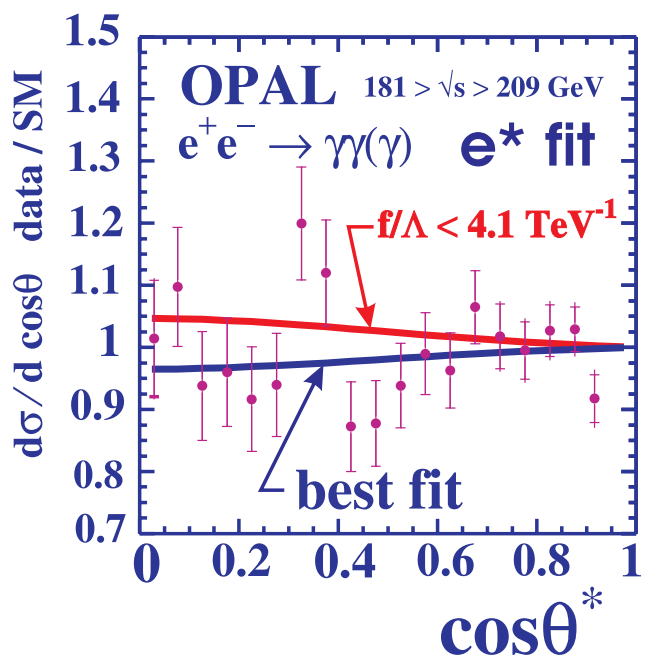

Figure 2. OPAL data for the cross-section measurement of the process $\mathrm{e}^{+} \mathrm{e}^{-} \rightarrow \gamma \gamma$ are shown by the points with error bars. Also shown is a fit assuming the presence of an excited electron assuming $f=f^{\prime}$.

fractions of the different LQ types to either a quark and a charged lepton or a quark and a neutrino. The coupling of the LQ to each quark and lepton (or neutrino) is parametrized with a Yukawa coupling $\lambda$. Constraints on leptoquarks exist both from direct searches at colliders, and from processes which are rare in, or forbidden by, the Standard Model. In particular, leptoquarks with quantum numbers from different fermion generations are severely constrained by FCNC and lepton flavour violation searches in $\mathrm{K}$ and $\mathrm{B}$ meson decays.

Leptoquarks could be observed directly as a peak in the lepton-jet mass spectrum in a process such as ep $\rightarrow$ LQ X with LQ $\rightarrow$ eq (where the quark hadronizes to form a jet). An example of a search from H1 is shown in Figure 4.

Leptoquarks could also be observed indirectly by their effect on Standard Model processes. For example, $t$-channel leptoquark exchange would change the observed cross-section of the process 


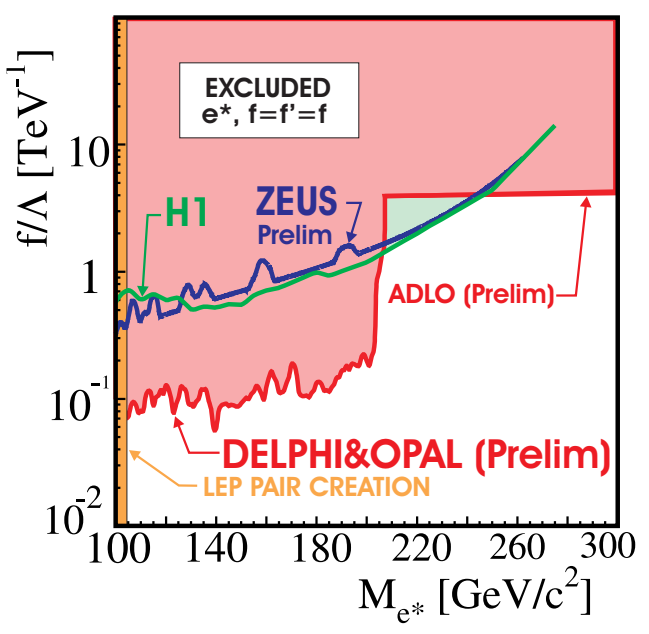

Figure 3. Constraints from LEP and HERA on excited electron masses and couplings assuming $f=f^{\prime}$. The LEP experiments exclude masses below about $103 \mathrm{GeV}$ from searches for pair creation, while both LEP and HERA place contraints on the ratio of the coupling and the compositeness scale from searches from single production. The LEP experiments also constrain this ratio to higher masses using the process $\mathrm{e}^{+} \mathrm{e}^{-} \rightarrow \gamma \gamma$.

$\mathrm{e}^{+} \mathrm{e}^{-} \rightarrow \mathrm{q} \overline{\mathrm{q}}$. Results of a fit to the differential cross-section of this process from ALEPH are shown in Figure 5 [11].

Combining all constraints on Leptoquarks and assuming as an example a LQ fermion number $\mathrm{F}=2$, constraints on the LQ couplings are shown in Figure 6. There is a direct constraint on the LQ mass from the search for the process gluon $\rightarrow$ LQ LQ from the Tevatron experiments. For larger masses, searches from HERA and LEP constrain the coupling for different LQ masses.

\subsection{Anomalous top quark FCNC cou- plings}

Many models of physics beyond the Standard Model predict anomalous couplings among the fermions, and in particular could have tree-level flavour changing neutral currents, FCNC. While precise measurements of quark and lepton prop-

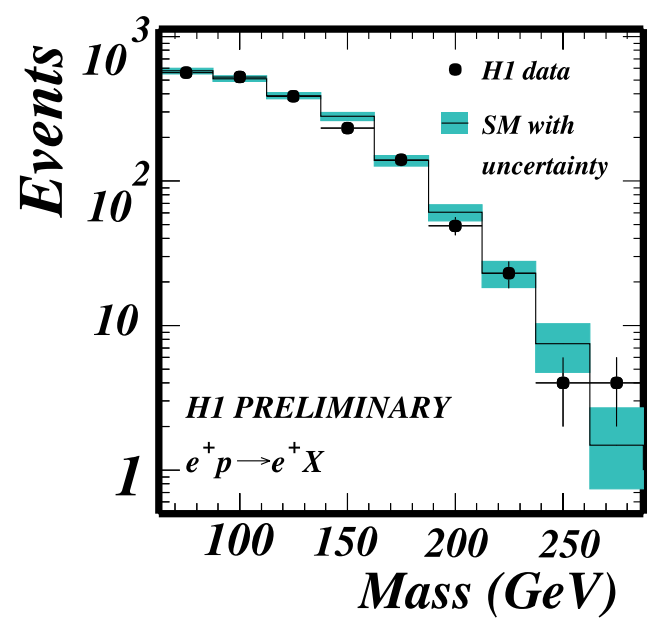

Figure 4. The electron-jet invariant mass from the direct search for leptoquarks from H1. The points are the $\mathrm{H} 1$ data, and the solid lines are the expectation from the Standard Model with the errors indicated by the shaded bands.

erties exclude such effects for most fermions, the top quark couplings remain relatively poorly measured. Anomalous FCNC top quark couplings could cause large branching fractions for decays such as $\mathrm{t} \rightarrow \mathrm{c}(\mathrm{u}) \mathrm{Z}$ or $\mathrm{t} \rightarrow \mathrm{c}(\mathrm{u}) \gamma$. The strengths of the anomalous top quark couplings are commonly parametrized in terms of couplings $\kappa_{\mathrm{Z}}$ and $\kappa_{\gamma}[12]$.

If such couplings exist, it would also be possible to produce top quarks singly at LEP or HERA via processes such as $\mathrm{e}^{+} \mathrm{e}^{-} \rightarrow \mathrm{t} \mathrm{c}(\mathrm{u})$ (LEP) and e $\mathrm{u} \rightarrow \mathrm{e} \mathrm{t}$ (HERA). The top quark would then be expected to decay via $\mathrm{t} \rightarrow \mathrm{b} \mathrm{W}$, and also via exotic decay modes such as the hypothesized FCNC processes $\mathrm{t} \rightarrow \mathrm{c}(\mathrm{u}) \mathrm{Z}$ and $\mathrm{t} \rightarrow \mathrm{c}(\mathrm{u}) \gamma$.

A clean search channel at HERA is when the $\mathrm{W}$-boson from the $\mathrm{t} \rightarrow \mathrm{b} \mathrm{W}$ process decays leptonically, providing a signature of high transverse momentum leptons and missing energy. An example of the charged lepton transverse momentum spectrum for electrons and muons from $\mathrm{H} 1$ is shown in Figure 7, where an excess is observed 


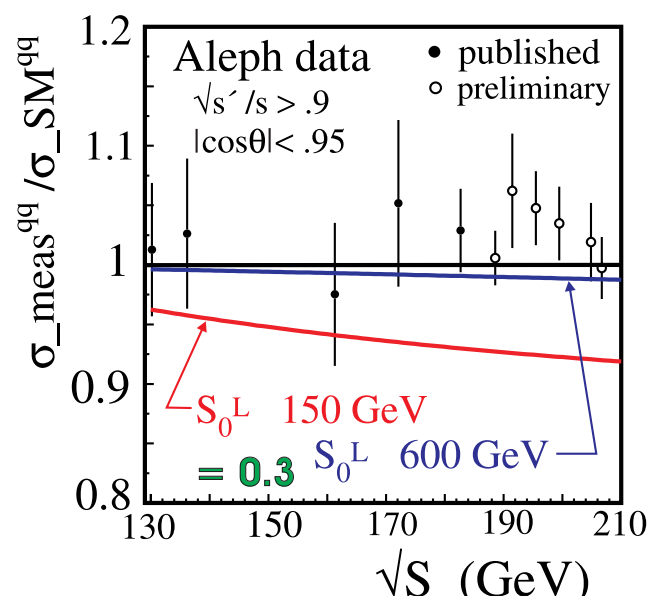

Figure 5. ALEPH e $\mathrm{e}^{+} \mathrm{e}^{-} \rightarrow \mathrm{q} \overline{\mathrm{q}}$ cross-section normalized to the Standard Model expectation, with fits to possible contributions from t-channel leptoquark exchange. The solid and empty circles are ALEPH published and preliminary data, respectively. The indicated curves are expectations in the presence of leptoquarks with a Yukawa coupling of 0.3 (approximately EM strength).

( 6 data events with $1.0 \pm 0.3$ expected background for the transverse momenta of the event recoiling against the lepton greater than $40 \mathrm{GeV}$ ) [13]. The ZEUS data, however, show good agreement between data and the expected background in the electron and muon channels. ZEUS does observe one tau candidate above the same $40 \mathrm{GeV}$ transverse momentum cut with $0.06 \pm 0.01$ background events expected [14]. Data from the ZEUS tau channel are shown in Figure 8.

Constraints on the couplings $\kappa_{\mathrm{Z}}$ and $\kappa_{\gamma}$ are shown in Figure 9 [15]. The constraints from CDF are direct translations of constraints on anomalous top-quark branching fractions. The LEP and HERA constraints are somewhat more model dependent, because the production cross-sections and detection efficiencies depend on the details of the FCNC model. The LEP constraints are valid for both $\mathrm{t} \rightarrow \mathrm{c}$ and $\mathrm{t} \rightarrow \mathrm{u}$, while the HERA constraints are valid only for $t \rightarrow u \gamma$.

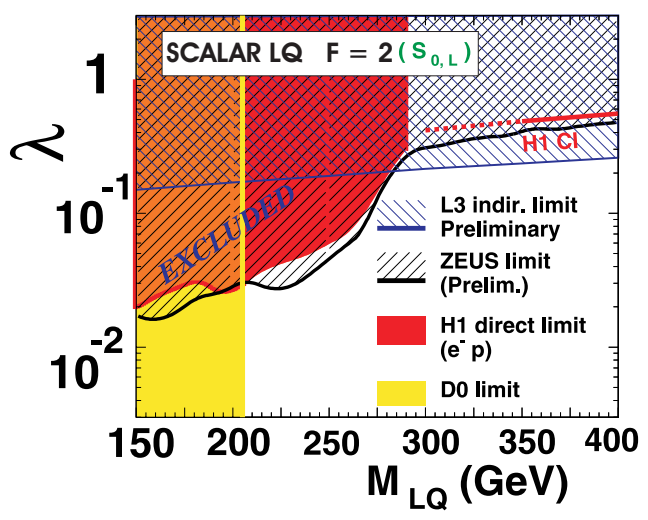

Figure 6. Example limits on the couplings for different masses of first generation leptoquarks. The $S_{0, L}$ LQ has charge $1 / 3$ and $\mathrm{BR}(\mathrm{LQ} \rightarrow$ eq $)=$ $1 / 2$.

\section{Supersymmetry}

Supersymmetry [16], where all SM particles have a partner whose spin differs by a half unit, provides an elegant method of solving the problem of fine-tuning the Higgs mass. In the minimal supersymmetric extension to the Standard Model, the MSSM, an additional 105 parameters are added to the SM [17], but the general model has many serious problems (eg. unsupressed FCNC). Many simpler models exist which are constructed to reduce the number of free parameters and to avoid the serious problems with the MSSM. The most popular are "top-down" models which assume a simple particle spectrum at the highest energy scales $\left(M_{\mathrm{GUT}}\right.$ or $M_{\text {Planck }}$ determined by a few parameters, and the electroweak scale particle masses and couplings can be determined from renormalization group evolution. One of the most popular models remains minimal supergravity (mSUGRA). An example mSUGRA particle spectrum for different energy scales is shown in Figure 10 (taken from [18]). In mSUGRA, the lightest SUSY particle (LSP) is typically the weakly interacting lightest neu- 


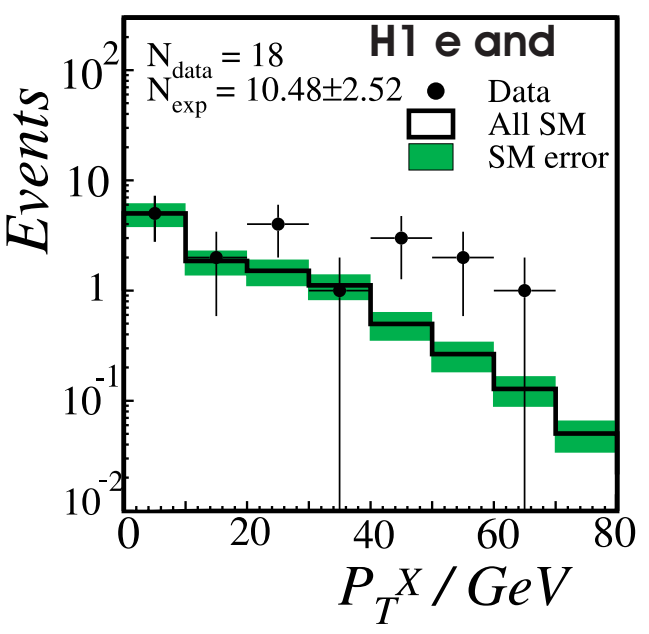

Figure 7. The transverse momentum of the system recoiling from the electron or muon in the H1 isolated lepton analysis. The points with error bars are the $\mathrm{H} 1$ data, and the solid line is the SM expectation with the error indicated by the shaded region.

$\operatorname{tralino}^{1}\left(\tilde{\chi}_{1}^{0}\right)$.

Another popular model is one in which SUSY breaking is communicated via SM gauge interactions, gauge mediated SUSY breaking (GMSB), which contrasts to the mSUGRA case where SUSY breaking is mediated by gravity. The chief phenomenological difference between mSUGRA and GMSB is the LSP, which in GMSB is almost always a light $(<1 \mathrm{GeV})$ gravitino, $\tilde{\mathrm{G}}$.

More exotic models, including the possibility of SUSY breaking being mediated by the superconformal anomaly [19] (AMSB) are not further considered here. Also, only R-parity conserving SUSY is reviewed. In this case, the LSP (either $\tilde{\chi}_{1}^{0}$ or $\tilde{\mathrm{G}}$ ) is stable, and all SUSY particle decay chains end in decays to the LSP which characteristically carries away missing energy from events.

While experiments attempt to perform the

\footnotetext{
${ }^{1}$ The four neutralinos are linear combinations of the SUSY partners of the photon, Z, and SUSY Higgs particles, the $\tilde{\gamma}, \tilde{\mathrm{Z}}, \tilde{\mathrm{h}}$ and $\tilde{\mathrm{H}}$.
}

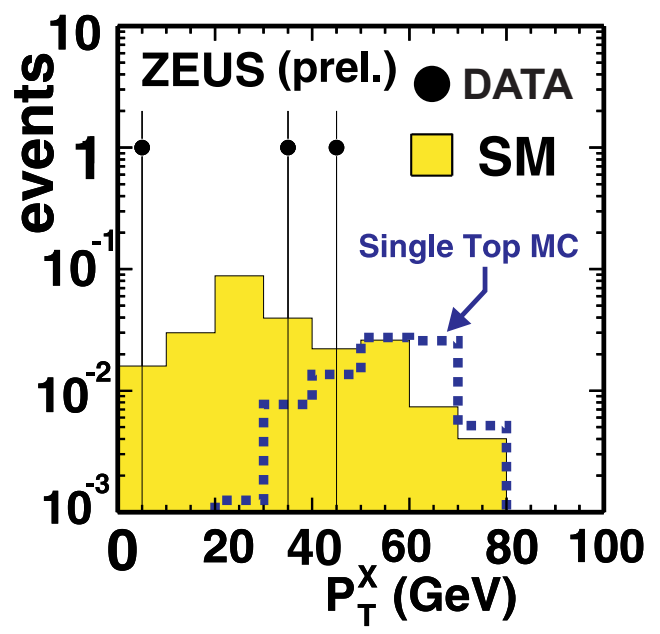

Figure 8 . The transverse momentum of the system recoiling from the tau-jet candidate in the ZEUS isolated tau lepton analysis. The points are the ZEUS data, and the shaded region indicates the SM expectation. The dashed line is the expectation from single top quark production (arbitrary normalization).

most model independent searches possible for SUSY signatures, the analyses are inevitably driven somewhat by the theoretical models. In particular, when no signal is observed, constraints are placed on the parameter space of the models.

\subsection{Gauge Mediated SUSY}

In GMSB models, all SUSY particles decay (sometimes cascading via other SUSY particles) to the next-to-lightest SUSY particle (NLSP), which then decays to the $\tilde{G}$ and the NLSP's partner SM particle. The NLSP is almost always either the $\tilde{\chi}_{1}^{0}$ or the lightest slepton $\left(\tilde{\ell}_{1}\right)$, leading to the decays ${ }^{2} \tilde{\chi}_{1}^{0} \rightarrow \tilde{\mathrm{G}} \gamma$ or $\tilde{\ell}_{1} \rightarrow \tilde{\mathrm{G}} \ell$. The coupling of the NLSP to the $\tilde{G}$ is suppressed, and the lifetime of the NLSP is effectively arbitrary. This leads to potentially interesting experimen-

\footnotetext{
${ }^{2}$ The lightest neutralino essentially always has a nonnegligible $\tilde{\gamma}$ component in GMSB, and the kinematically preferred $\gamma$ decay normally dominates over $\tilde{\chi}_{1}^{0} \rightarrow \tilde{\mathrm{G} Z}$ and $\tilde{\chi}_{1}^{0} \rightarrow \tilde{\mathrm{G}} \mathrm{h}$ (where $\mathrm{h}$ is the lightest SUSY Higgs boson).
} 


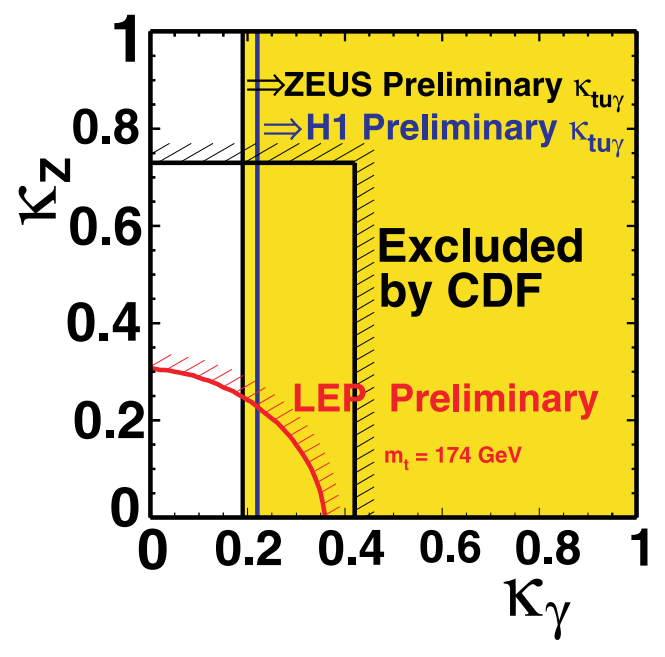

Figure 9. Excluded regions in the FCNC top quark couplings $\kappa_{\gamma}$ and $\kappa_{\mathrm{Z}}$ from CDF, H1, ZEUS and the combined LEP experiments. The HERA results are valid only for $\mathrm{t}-\mathrm{u}$ couplings.

tal signatures such as non-pointing photons from $\tilde{\chi}_{1}^{0}$ decays or kinked tracks (even heavy long lived charged particles) from the $\tilde{\ell}_{1}$.

A further complication in the $\tilde{\ell}_{1}$ NLSP case is that mixing in the third generation will preferentially make the NLSP the lightest scalar tau lepton $\left(\tilde{\tau}_{1}\right)$, particularly for large $\tan \beta(\approx 10)^{3}$. Example limits on the $\tilde{\tau}_{1}$ mass from searches at LEP [20] are shown in Figure 11. The limits for scalar muons are somewhat more restrictive, while the constraints on scalar electrons are rather model dependent due to the presence of $t$-channel $\tilde{\chi}^{0}$ exchange diagrams in the production process.

The many different particle searches are combined to constrain the parameter space allowed in a minimal GMSB model [21]. The most important parameters are $\tan \beta$ and the SUSY particle mass scale parameter, $\Lambda$. Example constraints from LEP are shown in Figure 12. The LEP constraints are comparable to those expected from Run IIa at the Tevatron, but the Tevatron should

${ }^{3} \tan \beta$ is the ratio of the vacuum expectation values of the two SUSY Higgs doublets, $v_{u} / v_{d}$.

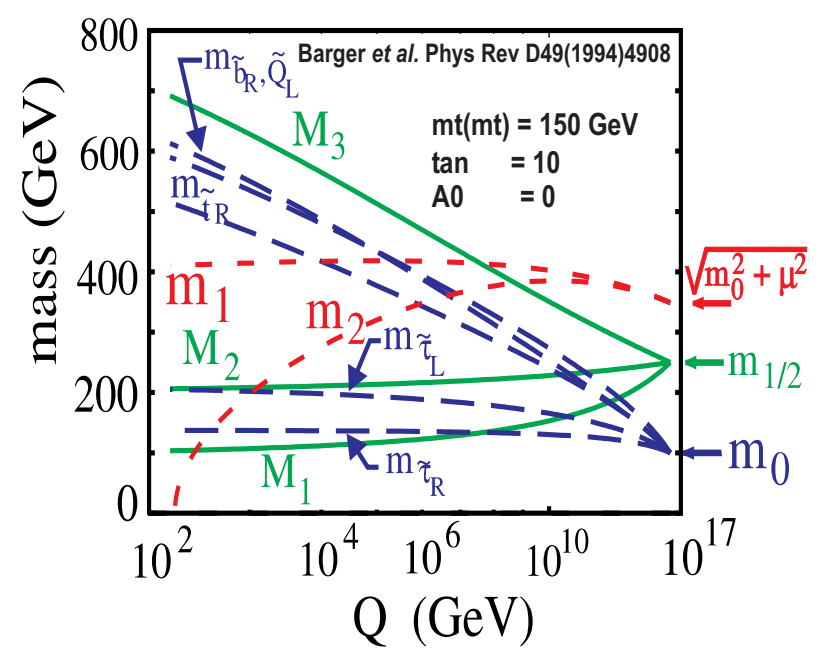

Figure 10. Example evolution of SUSY particle masses in mSUGRA from [18]. The parameter $m_{0}$ is the unified scalar particle mass at the GUT or Planck scale, $m_{1 / 2}$ is the gaugino mass at the GUT scale, and $\mu$ is the higgsino mass parameter. The additional parameters, the ratio of the higgs vevs $\tan \beta$ and the trilinear mixing parameter $A_{0}$, are fixed for this plot as indicated.

develop discovery sensitivity beyond LEP in the Run IIb era [22].

\subsection{Minimal SUGRA and related models}

Minimal SUGRA (mSUGRA) is the most common example model in which SUSY is mediated by gravity. With a $\tilde{\chi}_{1}^{0} \mathrm{LSP}$, the phenomenology is driven by the NLSP, which could in general be the next lightest neutralino $\left(\tilde{\chi}_{2}^{0}\right)$, the lightest chargino $^{4}\left(\tilde{\chi}_{1}^{ \pm}\right)$, the lightest slepton, or even in unusual configurations the lightest scalar squark $(\tilde{\mathrm{q}})$. The large top quark $(\tilde{\mathrm{t}})$ mass can cause the scalar top squark to be very light, and even be the NLSP.

Constraints on the masses of scalar quarks, assuming that all except for the $\tilde{t}$ are degenerate

\footnotetext{
${ }^{4}$ The two charginos are linear combinations of the SUSY partners of the $\mathrm{W}$ and charged Higgs boson, the $\tilde{W}$ and $\tilde{\mathrm{H}}^{ \pm}$
} 


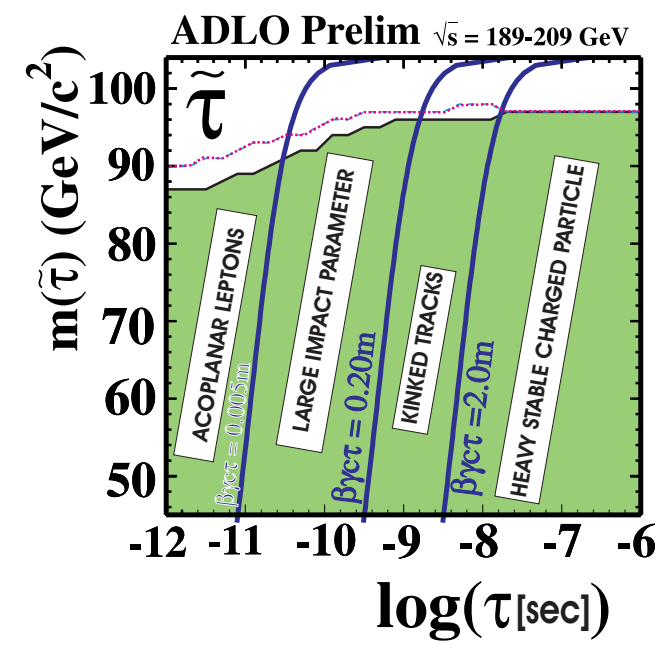

Figure 11. GMSB scalar tau exclusion region (shaded) for different lifetimes, assuming $\operatorname{BR}(\tilde{\tau} \rightarrow$ $\tau \tilde{\mathrm{G}})=1$. The median expected exclusion for a background only hypothesis is indicated with the dashed line. The solid lines are contours of different $\tilde{\tau}$ lifetimes (detector frame), which determine the most sensitive analysis channel.

in mass, from the Tevatron [23] and LEP [24] are shown in Figure 13. The limits assume GUT unification of the gluino and $\tilde{\chi}_{1}^{0}$ masses. Corresponding limits in the case of a $\tilde{t}$ NLSP are shown in Figure 14 [24]. The results from the CDF and D0 dominate the scalar quark sensitivity, except for small mass differences between the $\tilde{\mathrm{q}}$ and the $\tilde{\chi}_{1}^{0}$ where the LEP sensitivity is higher.

Combining the results from LEP searches for $\tilde{\chi}^{ \pm}, \tilde{\chi}^{0}, \tilde{\ell}, \tilde{\mathrm{q}}$ and Higgs bosons, constraints on parameters in the mSUGRA model are shown in Figures 15 and 16, which are projections in the $m_{1 / 2}$ vs. $m_{0}$ plane. Figure 15 shows the case $\tan \beta=50$ and $A_{0}=0$, and also includes projected sensitivities for direct searches from Run IIb, and also the contour corresponding to $B R\left(B_{S} \rightarrow \mu \mu\right)=10^{-7}$, which could be achievable even in Run IIa. Figure 16 shows the case $\tan \beta=30$, but allowing the $A_{0}$ parameter to have any value (which considerably decreases

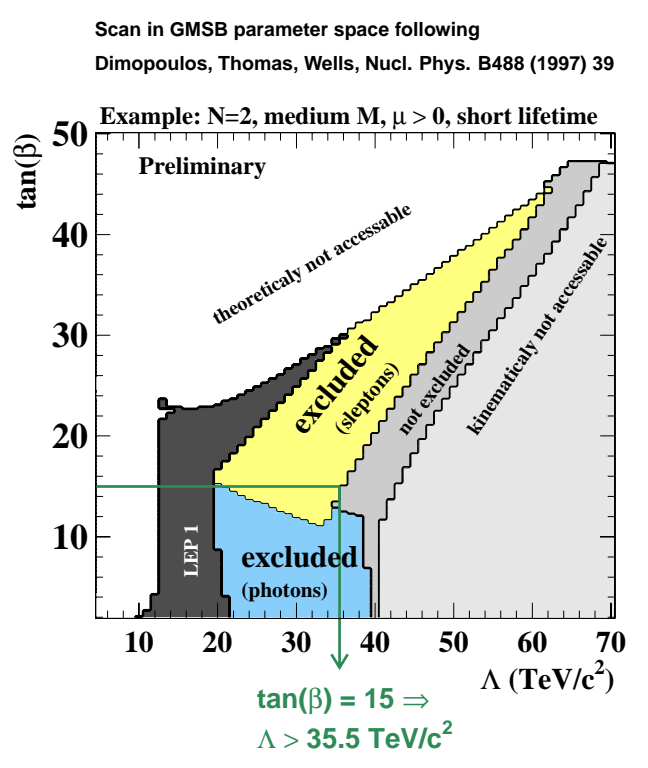

Figure 12. Example excluded region for GMSB in the $\tan \beta$ vs. $\Lambda$ (the SUSY particle mass scale parameter) plane from the combination of the data of the four LEP experiments.

the region excluded by LEP). Finally, Figure 17 shows excluded $\tilde{\chi}_{1}^{0}$ masses in the mSUGRA model as a function of $\tan \beta$. Masses less than $50 \mathrm{GeV}$ are excluded at the $95 \%$ confidence level for any value of the mSUGRA parameters.

\section{Non Standard Model Higgs particles}

\subsection{Decay mode independent Higgs search}

Models beyond the SM often have extended Higgs sectors, including SUSY. In many cases, there is at least one particle with properties close to the SM Higgs, although its branching ratios may be different. A very general search for the process $\mathrm{e}^{+} \mathrm{e}^{-} \rightarrow \mathrm{h} \mathrm{Z}$ has been presented by OPAL [26], which uses only the clean $\mathrm{Z}$ decay modes $\mathrm{Z} \rightarrow$ ee and $\mathrm{Z} \rightarrow \mu \mu$ to identify the events. The results are independent of the decay mode of the h. Excluded cross-sections from this search are 


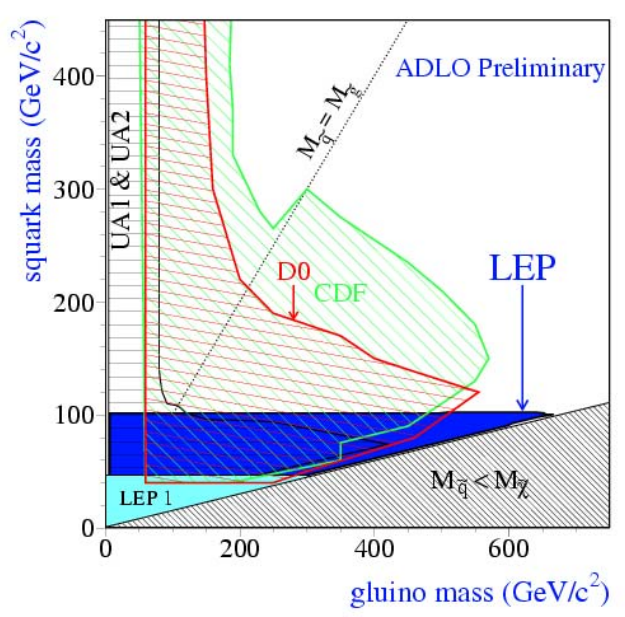

Figure 13. Excluded scalar quark and gluino mass regions in models with a $\tilde{\chi}_{1}^{0}$ LSP assuming GUT relations between the gluino and $\tilde{\chi}_{1}^{0}$ masses and 5 mass-degenerate quarks.

shown in Figure 18. A Higgs with a SM hZ crosssection is excluded at the $95 \%$ confidence level for masses less than $82 \mathrm{GeV}$.

Many other searches have been conducted that make different assumptions about the decays of the Higgs bosons, including so-called "flavourindependent" searches where the Higgs is required to decay into any pair of SM fermions. In that case, masses up to $113 \mathrm{GeV}$ have been excluded by the LEP experiments [27].

\subsection{Fermiophobic Higgs}

The critical feature of the Higgs boson as a mechanism for electroweak symmetry breaking is its coupling to the vector bosons, and models have been constructed that allow only these couplings and no coupling to SM fermions, a "fermiophobic" Higgs. For masses less than about $100 \mathrm{GeV}$, the decay $\mathrm{h} \rightarrow \gamma \gamma$ (which proceeds via a $\mathrm{W}$ loop) has the largest branching ratio. Upper limits at the $95 \%$ confidence level on the branching ratio for $\mathrm{h} \rightarrow \gamma \gamma$ from LEP are shown in Figure 19 [28]. For the branching ratio expected in a sim-

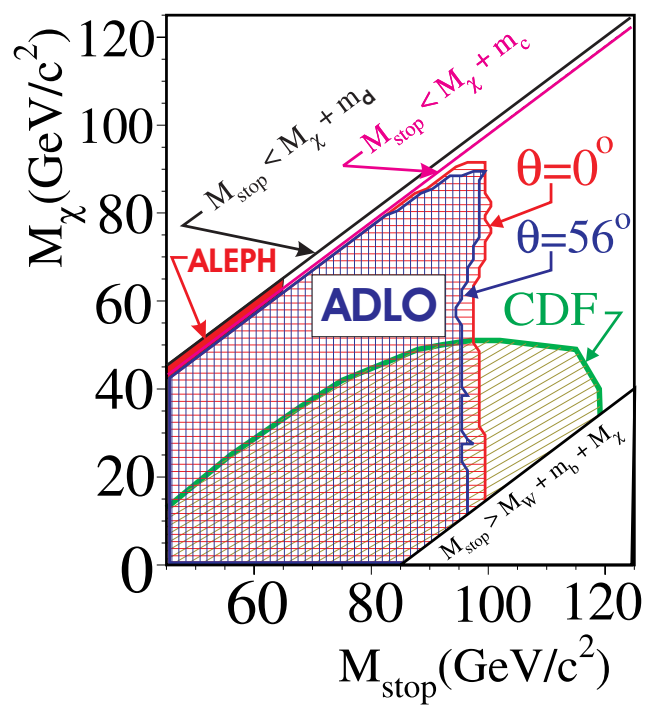

Figure 14. Excluded scalar top and $\tilde{\chi}_{1}^{0}$ masses from combinations of the four LEP experiments (ADLO) and from CDF.

ple Fermiophobic Higgs model, masses less than $110 \mathrm{GeV}$ are excluded at the $95 \%$ confidence level.

\subsection{Doubly-charged Higgs}

More exotic extended Higgs sectors, such as those contained in Left-Right Symmetric models, can contain doubly-charged Higgs bosons $\left(\mathrm{H}^{ \pm \pm}\right.$ or $\left.\Delta^{ \pm \pm}\right)$. They are called left or right handed depending on the helicity of the particles they couple to. Searches have been conducted for the pair creation of $\mathrm{H}^{ \pm \pm}$by DELPHI [29] and OPAL [30], yielding $95 \%$ confidence level mass limits of about $100 \mathrm{GeV}$.

An $\mathrm{H}^{ \pm \pm}$could also be singly produced at HERA or LEP. The decay signature would be the striking one of like-signed lepton pairs with a peak in their invariant mass spectrum. An excess was observed by H1 [31] in events with two or three $\mathrm{e}^{ \pm}$(denoted electrons), with the invariant mass of one pair of electrons above $100 \mathrm{GeV}$. This invariant mass distribution is shown in Figure 20 for 2-electron final states and in Figure 21 for 


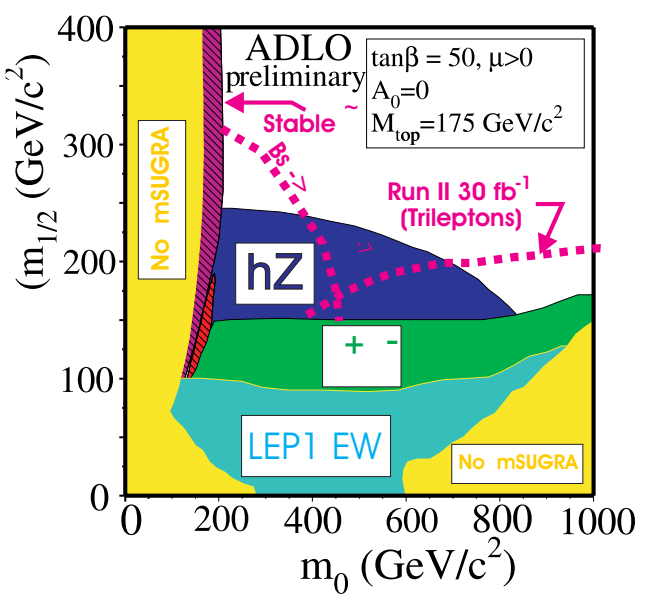

Figure 15. Regions excluded at the $95 \%$ confidence level from the LEP experiments from different search processes in the mSUGRA model. Also indicated is the contour corresponding to $B R\left(B_{S} \rightarrow \mu \mu\right)=10^{-7}$ which may be measurable at Run IIa at the Tevatron, and also the reach from direct searches at the Tevatron with the full Run IIb data set in the trilepton channel. The Tevatron prospects are taken from [25].

3-electron final states. ZEUS has performed similar analyses [32], but observed no excess. The dielectron invariant mass distribution from ZEUS is shown in Figure 22. While the H1 excess was initially reported in the context of possible hints of a doubly-charged Higgs boson, additional analysis from H1 does not support that hypothesis [34].

OPAL has also performed a search for the single production of doubly-charged Higgs bosons [35]. Their selection is sensitive to all lepton flavour combinations, and uses the well-constrained $\mathrm{e}^{+} \mathrm{e}^{-}$ collision system to reconstruct the di-lepton invariant mass even for the $\mathrm{H}^{ \pm \pm} \rightarrow \tau^{ \pm} \tau^{ \pm}$mode. This invariant mass distribution is shown in Figure 23, and no excess is observed. OPAL has also constrained the coupling of $\mathrm{H}^{ \pm \pm}$to electrons by fitting their measured Bhabha scattering differential cross-section assuming the presence of $t$-channel $\mathrm{H}^{ \pm \pm}$exchange, shown in Figure 24 .

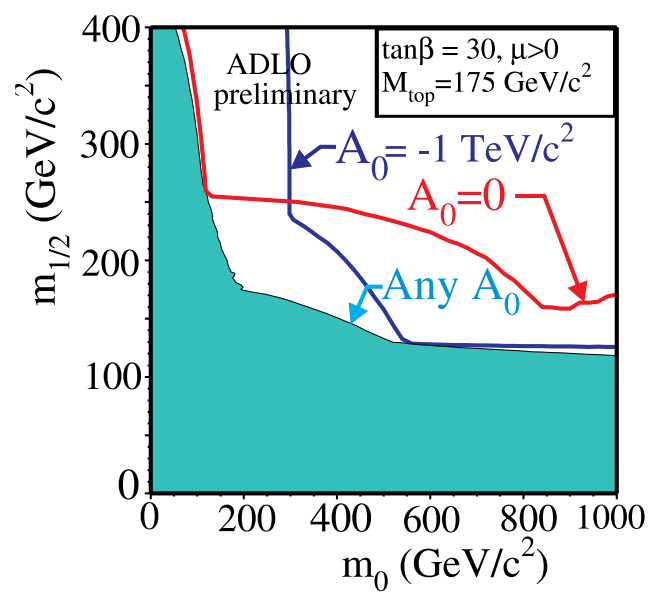

Figure 16. Regions excluded at the 95\% confidence level by the LEP experiments from different search processes in the mSUGRA model, but allowing the parameter $A_{0}$ to take any value.

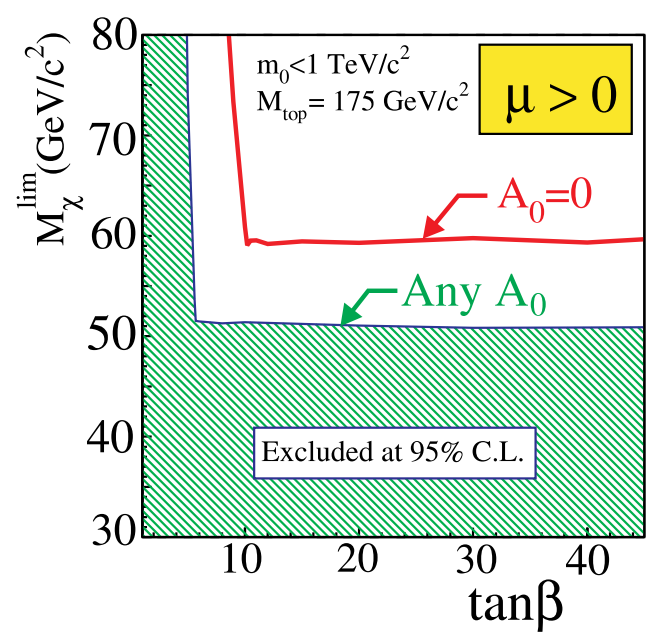

Figure 17. Masses of the LSP $\tilde{\chi}_{1}^{0}$ excluded at the $95 \%$ confidence level by the LEP experiments in the mSUGRA model. 


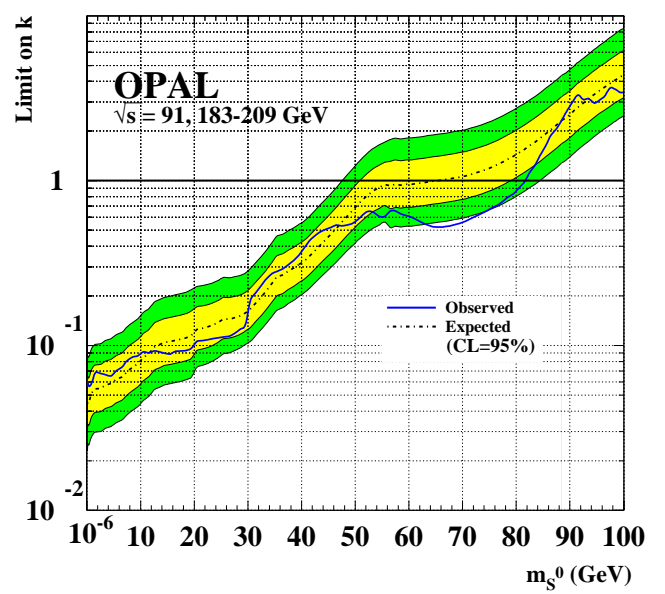

Figure 18. Excluded cross-sections (95\% C.L.) for the process $\mathrm{e}^{+} \mathrm{e}^{-} \rightarrow \mathrm{h} \mathrm{Z}$ followed by any decay of the $\mathrm{h}$ from OPAL. The solid line is the observed limit, while the dashed line (surrounded by $1-\sigma$ and $2-\sigma$ bands) is the median expected limit assuming a background-only hypothesis. The y-axis is the cross-section normalized to the SM HZ expected cross-section.

The different constraints on $\mathrm{H}^{ \pm \pm}$from $\mathrm{H} 1$, DELPHI and OPAL are shown in Figure 25. Any interpretation of the $\mathrm{H} 1$ excess in terms of $\mathrm{H}^{ \pm \pm}$production is thoroughly excluded by the OPAL results. Figure 26 shows the exclusion from OPAL to higher masses, where significant sensitivity to the coupling of the $\mathrm{H}^{ \pm \pm}$to electrons is maintained even above $1 \mathrm{TeV}$.

\section{Low scale gravity in extra dimensions}

Extra dimensions beyond our usual four have been considered in many models of new physics, including string theory. The relatively new idea that at least some of these extra dimensions could be large offers an attractive possibility to solve the problem of the apparent hierarchy of energy scales by reducing the scale of gravity to the $\mathrm{TeV}$ range. There are several different classes of models with large extra dimensions, and most experimental re-

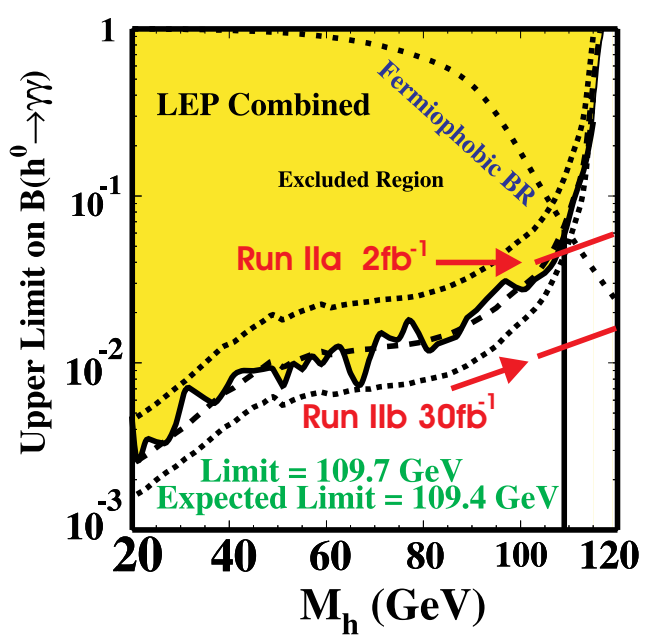

Figure 19. The 95\% confidence level excluded region for the branching ratio $\mathrm{h} \rightarrow \gamma \gamma$, assuming the SM cross-section for the process $\mathrm{e}^{+} \mathrm{e}^{-} \rightarrow \mathrm{h} \mathrm{Z}$, from the LEP experiments. The shaded region indicates the excluded region, while the dashed line indicates the expected limit in the background only hypothesis. The dotted line is the $\mathrm{h} \rightarrow \gamma \gamma$ branching ratio in a simple fermiophobic Higgs model where the Higgs coupling to fermions is set to zero.

sults have been been presented in the context of the framework from Arkani-Hamed, Dimopoulos and Dvali (ADD) [36]. In these models, gravity has a scale $M_{D}$ (presumably near $1 \mathrm{TeV}$ ) and propagates in $N$ extra dimensions with size $R$. While each graviton, G, has unobservably weak interactions, experimental observables may nevertheless be affected because processes are sensitive to a tower of Kaluza-Klein excitations of the graviton, $\mathrm{G}_{\mathrm{KK}}$. Assuming $M_{D} \sim 1 \mathrm{TeV}, N=1$ is excluded because it would imply a breakdown of Newtonian gravity at distances $\mathcal{O}\left(10^{11}\right) \mathrm{m}$.

Indirect effects from $\mathrm{G}_{\mathrm{KK}}$ exchange could be observed from their interference in SM processes such as $\mathrm{e}^{+} \mathrm{e}^{-} \rightarrow \mathrm{e}^{+} \mathrm{e}^{-}$via both $s$-channel and $t$-channel graviton exchange. It is not currently possible to calculate fully the effects of virtual 


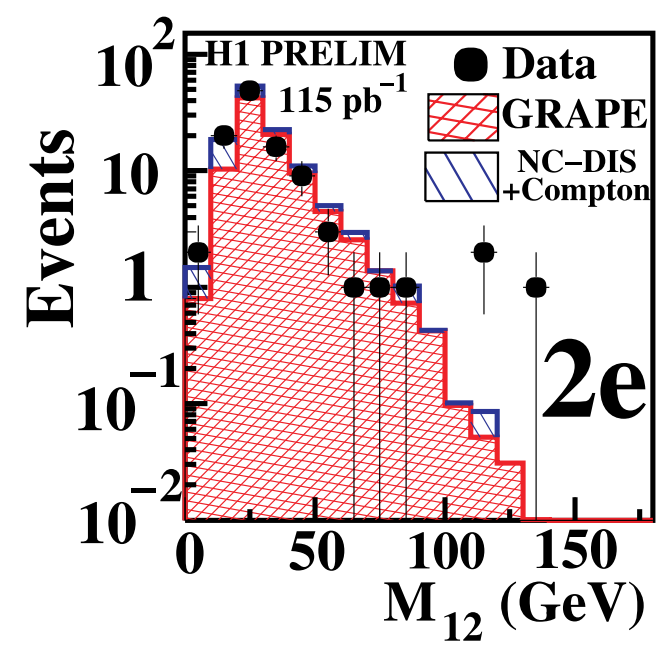

Figure 20. The invariant mass of the two electrons from $\mathrm{H} 1$ in their 2 isolated electron analysis. The points are the $\mathrm{H} 1$ data, and the shaded region is the expectation from SM processes. H1 observes 3 events with $0.25 \pm 0.05$ expected from $\mathrm{SM}$ processes with masses above $100 \mathrm{GeV}$.

$\mathrm{G}_{\mathrm{KK}}$ exchange on such processes due to ultraviolet (UV) divergences. Instead, a UV cutoff is introduced, called $\Lambda$ or $M_{S}$, to regularize the calculation, which is presumably of the order of $M_{D}$. There are several different formalisms used for the calculations, varying in degrees of model dependence. Here, the convention from Hewett [37] is adopted, which makes minimal assumptions about the details of the extra dimensions. Results of a fit to the differential Bhabha Scattering cross-section from the four LEP experiments to the cut-off parameter $M_{S}$ from [37] are shown in Figure 27 [38]. $M_{S}<1 \mathrm{TeV}$ is strongly excluded.

Graviton emission at accelerators could be observed in processes such as $\mathrm{q} \overline{\mathrm{q}} \rightarrow \mathrm{g} \mathrm{G}_{\mathrm{KK}}$ (where $\mathrm{g}$ is a gluon) or $\mathrm{e}^{+} \mathrm{e}^{-} \rightarrow \gamma \mathrm{G}_{\mathrm{KK}}$. In this case, constraints can be set directly on $M_{D}$ for different $N$. Constraints from DELPHI on the cross-section for $\mathrm{e}^{+} \mathrm{e}^{-} \rightarrow \gamma \mathrm{G}_{\mathrm{KK}}$ for different $N$ are shown in Figure 28, along with $95 \%$ confidence level limits

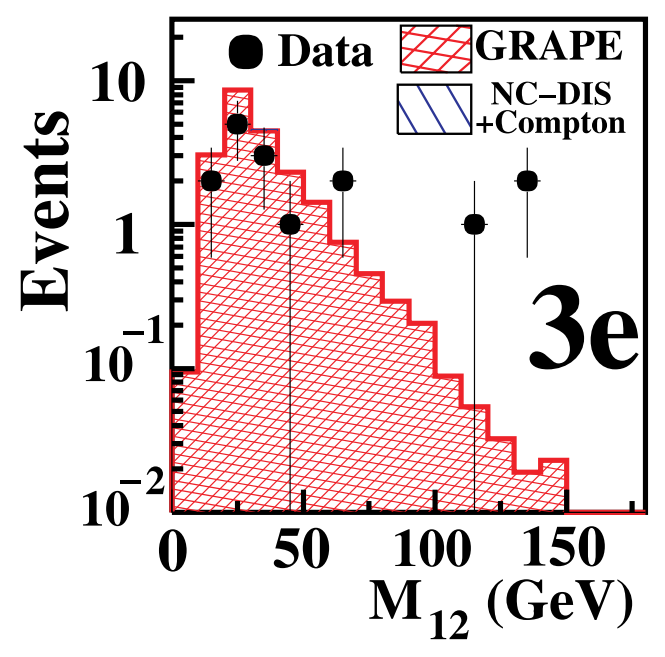

Figure 21. The invariant mass of the two most energetic electrons from $\mathrm{H} 1$ in their 3 isolated electron analysis. The points are the $\mathrm{H} 1$ data, and the shaded region is the expectation from SM processes. H1 observes 3 events with $0.23 \pm 0.04$ expected from SM processes with masses above $100 \mathrm{GeV}$.

on $M_{D}$.

\section{Non-commutative QED}

Theories with extra dimensions, such as string theory, can also naturally contain noncommutative geometry with a "preferred direction" in space. The scale of these noncommutative effects, $\Lambda_{N C}$, would be the scale of the extra dimensions, and in the case of low-scale extra dimensions the preferred direction could be observable in collider experiments. While no full non-commutative version of the Standard Model exists, there are consistent models of non-commutative quantum electrodynamics (QED) [39]. OPAL has considered possible non-commutative effects in the QED process $\mathrm{e}^{+} \mathrm{e}^{-} \rightarrow \gamma \gamma$ [40]. Particularly striking effects could appear in the detector-level azimuthal differential cross-section, $\mathrm{d} \sigma / \mathrm{d} \phi$, or even more con- 


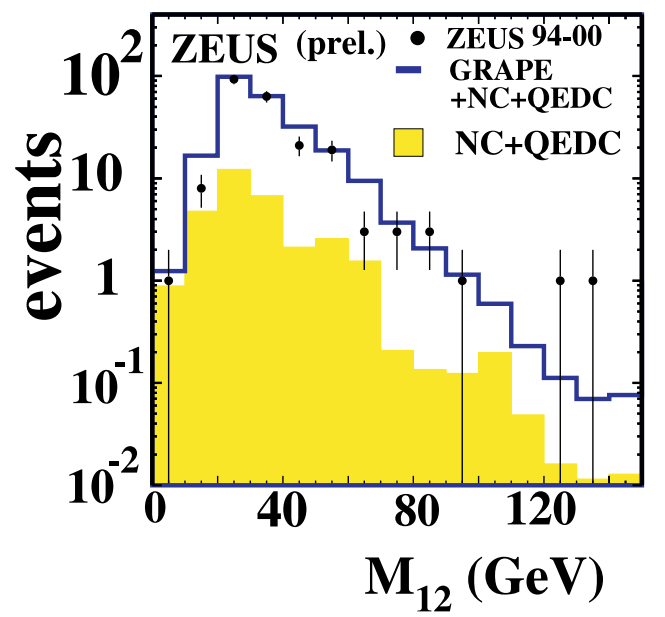

Figure 22. The invariant mass of the two most energetic electrons from ZEUS in their 2 or 3 isolated electron analysis. The points are the ZEUS data, and the shaded region is the expectation from SM processes. ZEUS observes 2 events with $1.14 \pm 0.09$ expected from SM processes with masses above $100 \mathrm{GeV}$.

vincingly in a time (or rotation angle) corrected differential cross-section which removes the effect of the motion of earth by projecting the detector coordinates into those of the preferred frame. Fits of these two differential cross-sections are shown in Figures 29 and 30, respectively. OPAL excludes $\Lambda_{N C}<142 \mathrm{GeV}$ at the $95 \%$ confidence level for any preferred direction [40].

\section{Conclusions}

The field of searches for physics beyond the Standard Model is a rich one, and the many experimental measurements constrain possible new physics. To date, there is no convincing evidence for physics beyond the Standard Model. The most comprehensive constraints on models such as SUSY, exotic Higgs, compositeness, heavy vector bosons, and many other varieties of new physics, come from LEP, the Tevatron Run I, and HERA I. Indeed, many of the most

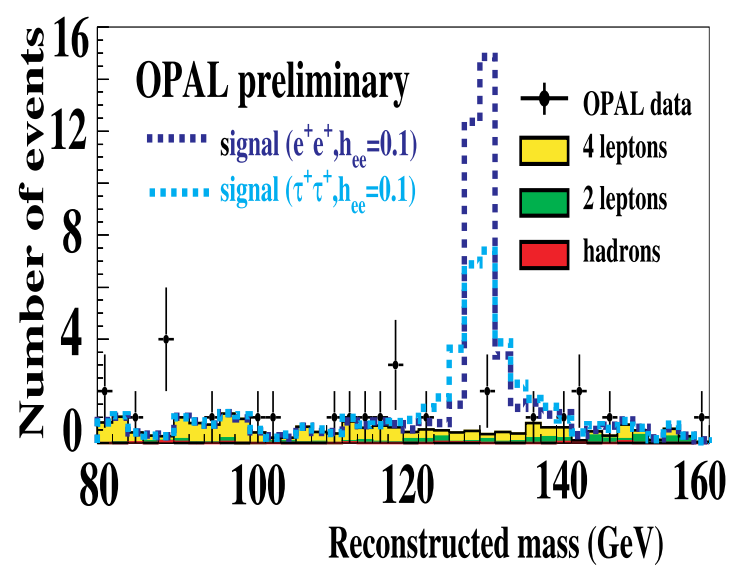

Figure 23. The reconstructed di-lepton mass from OPAL in their doubly-charged Higgs direct search. The points are the OPAL data, while the shaded region is the expectation from SM processes. The dashed lines are the expectations from a doubly-charged Higgs with a Yukawa coupling of 0.1 .

severe constraints come from the four LEP experiments, and many of the final results from ALEPH, DELPHI, L3 and OPAL were presented at ICHEP2002. The LEP era is, however, coming to an end as combinations of the four experiments' data sets are finalized.

The next decade will be an exciting one, with much higher luminosities from the Tevatron Run II and HERA II, and then with the data from the LHC and a high energy linear $\mathrm{e}^{+} \mathrm{e}^{-}$collider such as TESLA probing at, and beyond, the TeV scale. With these data, we can look forward to definitively answering the question "Is there $\mathrm{TeV}$ scale new physics?" I look forward to the answer to that question being yes.

\section{Acknowledgements}

The scope of the subject of experimental results beyond the Standard Model is enormous, and it would have been impossible to cover even a small part of it without assistance from 


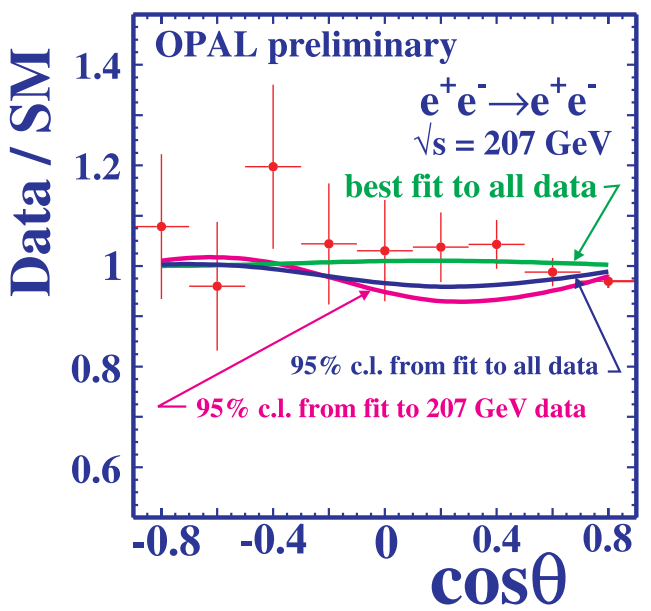

Figure 24. The OPAL Bhabha Scattering crosssection normalized to the SM expectation. The points are the OPAL data, while the lines are fits to, or limits on, a doubly-charged Higgs hypothesis.

many colleagues, both experimentalists and theorists. I would particularly like to mention the valuable contributions of Wolfgang Adam, Herbert Dreiner, Elisabetta Gallo, Geraldo Ganis, Steve Godfrey, Corinne Goy, Richard Hemingway, John Holt, Pat Kalyniak, Greg Landsberg, Paul Richard Newman, Mark Oreglia, David Plane, Christoph Rembser, Kirsten Sachs, André Schöning, Roberto Techini, Isabel Trigger, and Brigitte Vachon.

\section{REFERENCES}

1. E. Eichten, K. Lane, M. Peskin, Phys. Rev. Lett. 50 (1983) 811.

2. F. Boudjema,A. Djouadi, J.L. Kneur Z. Phys. C57 (1993) 425.

3. K. Hagiwara, D. Zeppenfeld, S. Komamiya Z. Phys. C29 (1985) 115.

4. ZEUS Collaboration,

"Search for excited fermions in ep collisions at HERA"

Paper \#607 contributed to International

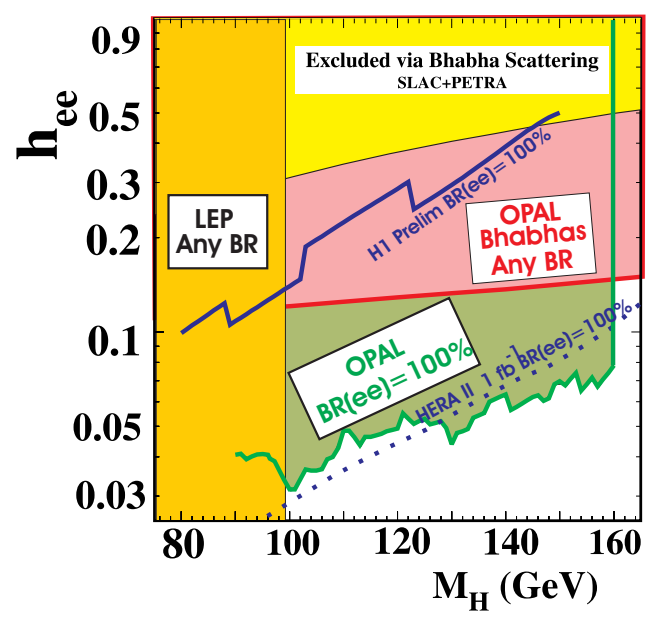

Figure 25. Limits from OPAL, DELPHI and H1 on the couplings and masses of a doubly-charged Higgs. Also shown with the dotted line is the expected sensitivity of $\mathrm{H} 1$ with the full HERA II data set.

Conference on High Energy Physics, Budapest, Hungary, 2001.

5. B. Vachon,

"Excited electron contribution to the $\mathrm{e}^{+} \mathrm{e}^{-} \rightarrow$ $\gamma \gamma$ cross-section," hep-ph/0103132, 2001.

6. OPAL Collaboration, G. Abbiendi et al., "Multi-Photon Production in e+e- Collisions at 181-209 GeV" CERN-EP-2002-060, accepted by Eur Phys J C.

7. DELPHI Collaboration,

"Search for excited leptons with the DELPHI detector at LEP,"

DELPHI note number: 2002-062-CONF-596, contributed to XXXIst International Conference of High Energy Physics, 24-31 July 2002, Amsterdam, The Netherlands.

8. OPAL Collaboration, G. Abbiendi et al., Phys. Lett. B544 (2002) 57.

9. LEP Exotica Working Group

"Search for excited leptons: preliminary combined results of the LEP experiments," 


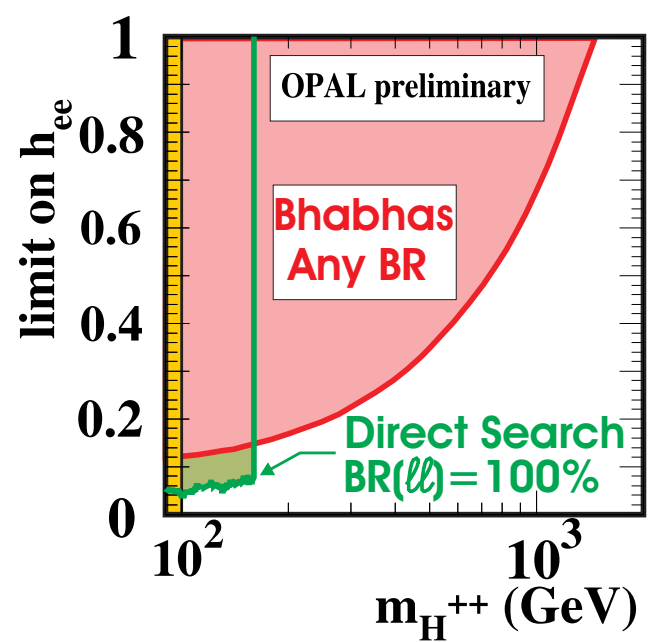

Figure 26. Region excluded at the 95\% C.L. from OPAL in the coupling vs. mass plane for a doubly-charged Higgs.

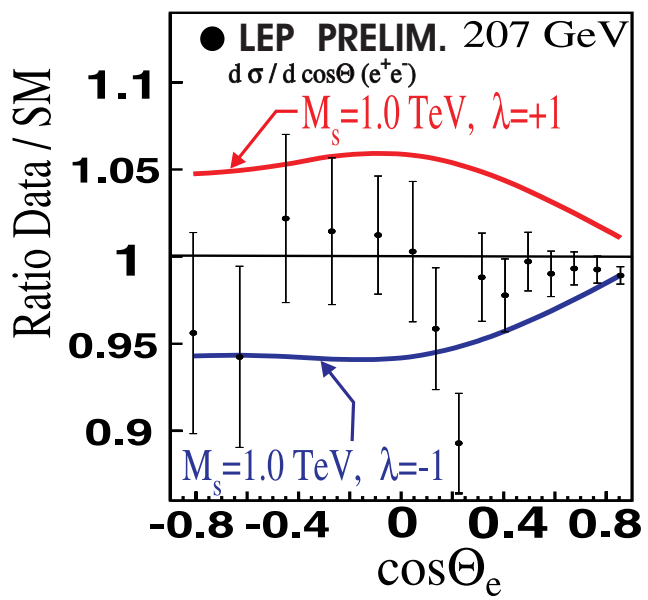

Figure 27. Fit to the combined LEP data to the Bhabha Scattering cross-section assuming the presence of low scale gravity in extra dimensions.

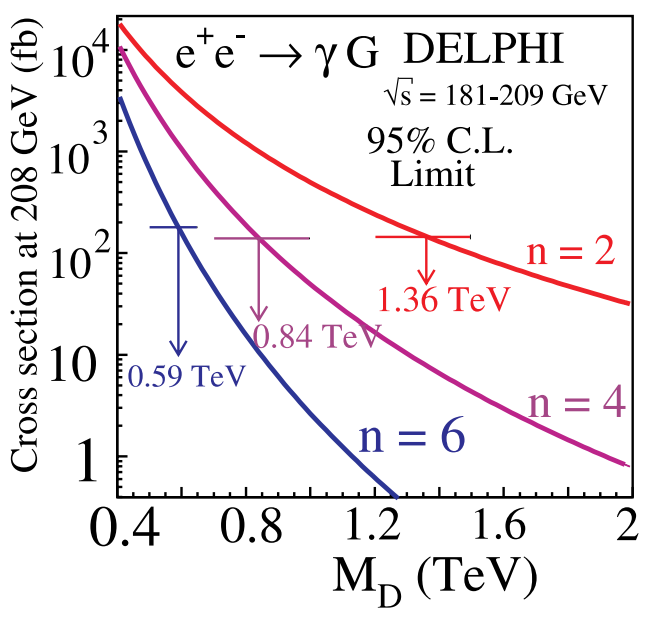

Figure 28. Limits at the $95 \%$ confidence level from DELPHI on the $\mathrm{e}^{+} \mathrm{e}^{-} \rightarrow \gamma \mathrm{G}$ cross-section in models with low scale gravity in extra dimensions for different numbers of extra dimensions. Also indicated are the inferred limits on the true Planck mass, $M_{D}$.

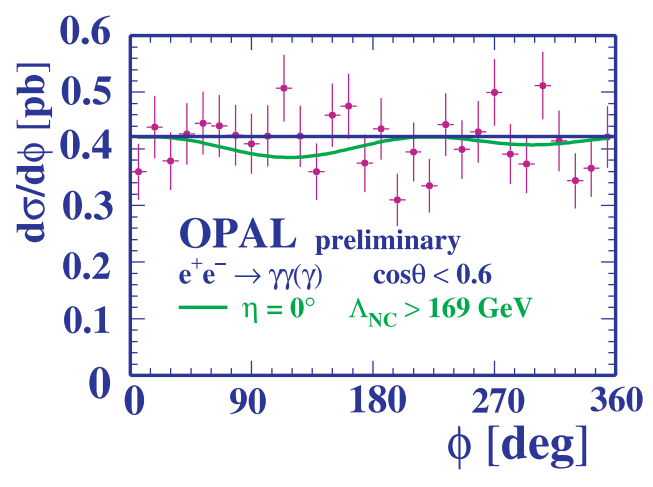

Figure 29. OPAL differential cross-section for the process $\mathrm{e}^{+} \mathrm{e}^{-} \rightarrow \gamma \gamma$. The points are the data, the flat line the SM expectation, and the solid sinusoidal curve the expectation from an example non-commutative geometry parameter set. 


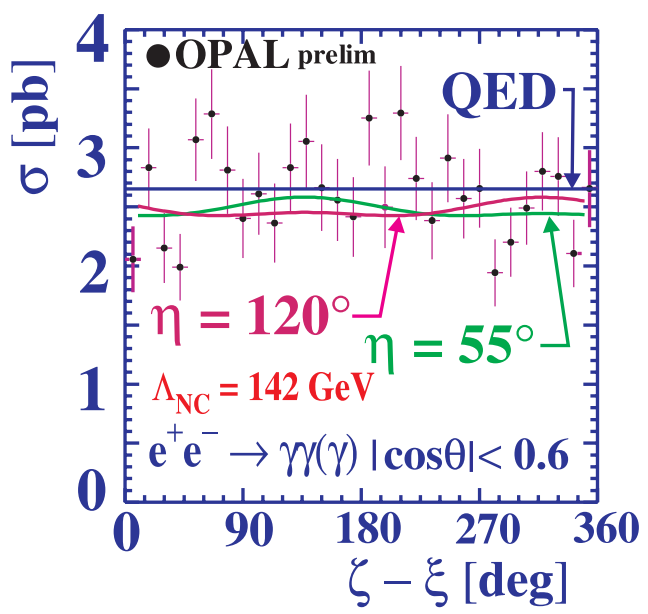

Figure 30. OPAL cross-section for the process $\mathrm{e}^{+} \mathrm{e}^{-} \rightarrow \gamma \gamma$ plotted $v s$. angle, corrected for the local motion of the earth in a presumed preferred reference frame in models with non-commutative geometry. The points are the OPAL data, the flat line the SM expectation, and the two sinusoidal curves the expectation from example noncommutative geometry parameter sets.

$\begin{array}{lccr}\text { LEP } & \text { Exotic WG } & \text { Note } & 2001- \\ 02, & \text { updated } & \text { July } & 2002\end{array}$ (http://lepexotica.web.cern.ch/LEPEXOTICA/).

10. W. Buchmüller, R. Rückl and D. Wyler, Phys. Lett. B191 (1987) 442.

11. ALEPH Collaboration,

"Fermion Pair Production in e+e- Collisions at High Energies and Limits on Physics beyond the Standard Model,"

ALEPH Note 2002-032, contributed to XXXIst International Conference of High Energy Physics, 24-31 July 2002, Amsterdam, The Netherlands.

12. J.F. Obraztsov, S. Slabospitsky, O. Yushchenko, Phys. Lett. B426 (1998) 393.

13. H1 Collaboration,

"Observation of isolated leptons with missing $P_{T}$ and comparison to $\mathrm{W}$ production at HERA"
Paper \#1022 contributed to XXXIst International Conference of High Energy Physics, 2431 July 2002, Amsterdam, The Netherlands.

14. ZEUS Collaboration,

"Study of multi-lepton production in ep interactions at HERA"

Paper \#910 contributed to XXXIst International Conference of High Energy Physics, 2431 July 2002, Amsterdam, The Netherlands.

15. LEP Exotica Working Group

"Search for single top production via flavour changing neutral currents: preliminary combined results of the LEP experiments,"

LEP Exotic WG Note 2001-01, (http://lepexotica.web.cern.ch/LEPEXOTICA/).

16. Y. Gol'fand and E. Likhtam, JETP Lett. 13 (1971) 323;

D. Volkov and V. Akulov, Phys. Lett. B46 (1973) 109;

J. Wess and B. Zumino, Nucl. Phys. B70 (1974) 39.

17. See, eg., H.E. Haber, Nucl.Phys.Proc.Suppl. 62 (1998) 469.

18. Barger et al. Phys. Rev. D49 (1994) 4908.

19. See, eg. G.F. Giudice, M. Luty, H. Murayama, R. Rattazzi, JHEP 98 (1998) 12.

20. LEP SUSY Working Group, ALEPH, DELPHI, L3 and OPAL experiments, note LEPSUSYWG/02-09.2 (http://lepsusy.web.cern.ch/lepsusy/Welcome.html)

21. S. Dimopoulos, S. Thomas, J. Wells, Nucl. Phys. B488 (1997) 39.

22. J. Quian et al., hep-ph/9903548 version 2 .

23. CDF Collaboration, T. Affolder et al., Phys. Rev. Lett. 88 (2002) 041801;

D0 Collaboration, B.Abbott et al., Phys. Rev. Lett. 83 (1999) 4937;

CDF Collaboration, T. Affolder et al., Phys. Rev. Lett. 84 (2000) 5704.

24. LEP SUSY Working Group, ALEPH, DELPHI, L3 and OPAL experiments, note LEPSUSYWG/02-02.1 (http://lepsusy.web.cern.ch/lepsusy/Welcome.html)

25. A. Dedes, H.K. Dreiner, U. Nierste, Phys. Rev. Lett. 87 (2001) 251804 and hepph/0207026.

26. OPAL Collaboration, G. Abbiendi et al., "Decay-mode independent searches for new 
scalar bosons with the OPAL detector at LEP"

CERN-EP-2002-032, submitted to Eur. Phys. $\mathrm{J}$ C.

27. LEP Higgs Working Group,

"Flavour Independent Search for Hadronically Decaying Neutral Higgs Bosons at LEP,"

LHWG Note/2001-07, July 2001.

28. LEP Higgs Working Group,

"Searches for Higgs Bosons Decaying into Photons: Combined Results from the LEP Experiments"

LHWG Note/2002-02, 12 July 2002.

29. DELPHI Collaboration,

"Search for doubly charged Higgs bosons," DELPHI note number 2002-039-CONF-573, contributed to XXXIst International Conference of High Energy Physics, 24-31 July 2002, Amsterdam, The Netherlands.

30. OPAL Collaboration, G. Abbiendi et al., Phys. Lett. B526 (2002) 221.

31. H1 Collaboration,

"Multi-electron Production at High Transverse Momentum in ep collisions at HERA"

Paper 1019, contributed to XXXIst International Conference of High Energy Physics, 2431 July 2002, Amsterdam, The Netherlands.

32. ZEUS Collaboration,

"Study of multi-lepton production in ep interactions at HERA"

Paper \#910 contributed to XXXIst International Conference of High Energy Physics, 2431 July 2002, Amsterdam, The Netherlands.

33. Y.Sirois,

" $R_{P}$ violating SUSY and exotics at HERA," plenary presentation at SUSY02: The 10th International Conference on Supersymmetry and Unification of Fundamental Interactions, 17-23 June 2002, DESY Hamburg.

34. H1 Collaboration,

"Search for doubly charged Higgs production at HERA"

Paper 1020, contributed to XXXIst International Conference of High Energy Physics, 2431 July 2002, Amsterdam, The Netherlands.

35. OPAL Collaboration,

"Search for the Single Production of Doubly-
Charged Higgs Bosons and Constraints on its Couplings from Bhabha Scattering,"

OPAL Physics Note PN502, contributed to XXXIst International Conference of High Energy Physics, 24-31 July 2002, Amsterdam, The Netherlands.

36. N. Arkani-Hamed, S. Dimopoulos and G. Dvali, Phys. Lett. B429 (1998) 263;

I. Antoniadis, N. Arkani-Hamed, S. Dimopoulos and G. Dvali, Phys. Lett. B436 (1998) 257 ;

N. Arkani-Hamed, S. Dimopoulos and G. Dvali, Phys. Rev. D59 (1999) 86004.

37. J.L. Hewett, Phys. Rev. Lett. 82 (1999) 4765.

38. LEP Electroweak Working

Group, note in preparation

(http://lepewwg.web.cern.ch/LEPEWWG/).

39. M. Hayakawa, Phys. Lett. B478 (2000) 394.

40. OPAL Collaboration,

"Test of non-commutative QED in the process $\mathrm{e}^{+} \mathrm{e}^{-} \rightarrow \gamma \gamma$ at LEP"

OPAL Physics Note PN500, contributed to XXXIst International Conference of High Energy Physics, 24-31 July 2002, Amsterdam, The Netherlands. 